\title{
Metabolomic Analysis on the Petal of 'Chen Xi' Rose with Light-Induced Color Changes
}

\author{
Mengyue Su ${ }^{1}$, Rebecca Njeri Damaris ${ }^{1}$, Zhengrong $\mathrm{Hu}^{1}{ }^{1}$, Pingfang Yang ${ }^{1, *}$ and Jiao Deng ${ }^{2, *}$ \\ 1 State Key Laboratory of Biocatalysis and Enzyme Engineering, School of Life Sciences, Hubei University, \\ Wuhan 430026, China; 201911110711029@stu.hubu.edu.cn (M.S.); njerirebecca09@gmail.com (R.N.D.); \\ huzhengrong1001@hubu.edu.cn (Z.H.) \\ 2 Research Center of Buckwheat Industry Technology, School of Life Sciences, Guizhou Normal University, \\ Guiyang 550001, China \\ * Correspondence: yangpf@hubu.edu.cn (P.Y.); ddj613@163.com (J.D.); Tel.: +86-27-88663882 (P.Y.); \\ +86-0851-86780649 (J.D.)
}

Citation: Su, M.; Damaris, R.N.; Hu, Z.; Yang, P.; Deng, J. Metabolomic Analysis on the Petal of 'Chen $\mathrm{Xi}^{\prime}$ Rose with Light-Induced Color Changes. Plants 2021, 10, 2065. https://doi.org/10.3390/ plants10102065

Academic Editors: Pablo F. Cavagnaro, Philipp W. Simon and Massimo Iorizzo

Received: 11 August 2021

Accepted: 27 September 2021

Published: 30 September 2021

Publisher's Note: MDPI stays neutral with regard to jurisdictional claims in published maps and institutional affiliations.

Copyright: (c) 2021 by the authors. Licensee MDPI, Basel, Switzerland. This article is an open access article distributed under the terms and conditions of the Creative Commons Attribution (CC BY) license (https:// creativecommons.org/licenses/by/ $4.0 /)$.

\begin{abstract}
Flower color is one of the most prominent traits of rose flowers and determines their ornamental value. The color of the "Chen $\mathrm{Xi}$ " rose can change from yellow to red during flower blooming. In the present study, the flavonoid metabolites were investigated by the UPLC-ESIMS/MS from the petals of four successive flower development stages under natural conditions. In total, 176 flavonoid components, including 49 flavones, 59 flavonols, 12 flavanones, 3 isoflavones, 12 anthocyanins, and 41 proanthocyanidins were identified, with some of them being detected for the first time in this study. Additionally, there were 56 compounds that showed differences among comparison groups, mainly being enriched in pathways of isoflavone, flavonoid, flavone, flavonol, phenylpropanoids, and anthocyanin. Among them, it is anthocyanins that allow the rose flower to turn red when exposed to sunlight. To verify this result, compounds from rose petal with shading treatment (S2D) was also detected but could be clearly separated from the four samples under light by clustering and principal component analyses (PCA). Consistent with low anthocyanins accumulation, the flower with shading could not turn red. Moreover, it provides a foundation for further research on the light-induced color modification of flower.
\end{abstract}

Keywords: rose flower; alteration color; flavonoid metabolites; anthocyanins; light induction

\section{Introduction}

The rose (Rosa sp.), belonging to the Rosaceae family, is one of the most popular and widely planted ornamental plants worldwide. It is also one of the top ten flowers in China, which is honored as the queen of flowers and has a long history of cultivation in China [1]. Roses are utilized as cut flowers, potted plants, and garden ornamental plants [2,3]. Due to their rich bioactive components, rose flowers are extensively used in food, drugs, cosmetics, and pharmaceutics [4-7]. Rose flowers are charming and colorful, including red, pink, orange, yellow, white, variegated, as well as alternating colors during flowering. Additionally, the blue rose flower has been cultivated through the molecular breeding method [8]. The color of the rose flower determines its ornamental and commercial value and creating novel flower colors is one of the main objectives for breeders.

It is known to all that plant color is determined by pigments, and there are four kinds of natural pigments that include flavonoids, carotenoids, chlorophyll, and alkaloids [9], which give color to plant flowers, leaves, vegetables, and fruits. Osterc [9] detected several phenolic compounds from rose petals of eight cultivars at four developmental stages, including five anthocyanins, which were regarded as the major factor for visual attributes of rose flower, and suggested that the total content of anthocyanin and quercetin (both belonging to flavonoids) continued accumulating from the bud stage until fully open flowers, then decreased in senescent ones. Previous studies indicated that in rose flowers, 
anthocyanins, determine the red color, carotenoids impart yellow color, while pink and orange colors are endowed by a combination of both anthocyanins and carotenoids, and nearly no pigments were detected in white flowers [10]. Lee et al. [11] identified two anthocyanins, cyanidin 3,5-di-O-glucoside and pelargonidin 3,5-di-O-glucoside in red rose flowers, with the former occupying $85 \%$ of total anthocyanins. Wan et al. [3] found nineteen flavonols and sixteen carotenoids in yellow petals of Rosa 'Sun City' cultivar, then they also detected four anthocyanins, 20 flavonols, and 10 carotenoids in the petals of six Rosa cultivars with yellow, pink, and orange color [10]. Combined with RNA-sequencing and metabolite analysis, Huang et al. [12] revealed the mechanism of flower color change in rose mutants. The results indicated that the expression levels of the differentially expressed genes enriched in the anthocyanin pathway, e.g., chalcone synthase (CHS), chalcone isomerase $(\mathrm{CHI})$, dihydroflavonol reductase (DFR), and leucoanthocyanidin dioxygenase $(L D O X)$ in pink flowers were significantly higher than in white flowers, which are consistent with the accumulation of anthocyanin in flowers of the two rose cultivars. By contrast, the expression of flavonol synthase (FLS) in the white rose flower was higher than that in red flowers, resulting in the accumulation of more flavonols. Therefore, these findings suggested that competition between anthocyanin and flavonol biosynthesis is a primary cause of rose flower color variation [12].

Light affects anthocyanin biosynthesis: for example, the anthocyanin pigmentation of some fruits' skin including red pears [13], apples [14], and grapes [15] is induced by light. In this study, we chose one rose cultivar whose flower color changed from yellow to red during development. Before the flower opens and the petal emerges from the calyx, the flower petals are yellow with an edge which, when exposed to the sun, become red, and after the flower totally opens and the petals are fully stretched, the red color intensifies with increased exposure to the sunlight. As the flower ages, the color of the petals slowly faded to white and wither at the end. However, when the flower fully opens and is moved to the shade, the color of the yellow petals persists and does not change to red and directly fades to white as it withers. To detect if the color change is induced by light, flavonoid metabolites of rose flowers at four developmental stages under natural conditions as well as one stage flower under shading treatment were determined. The results showed the dynamic change of flavonoid metabolites during the process of color change with the flower development and lay a theoretical foundation for further understanding of the mechanism underlying the light-induced rose flower pigmentation and ultimately facilitate breeding of rose cultivars with a novel flower color.

\section{Results}

\subsection{Light-Induced Color Changes of Rose Petals}

The 'Chen Xi' variety of rose has a very beautiful flower showing color changes during the blooming, which contributes a lot to its ornamental value. At the bud stage, sepals show a little red color in the green background and cover the whole flower (Figure 1A). After the sepals opened, petals on the surface showed some red color with a yellow background. When the flower was just fully opened, the petals were in yellow with a tiny amount of red at the edge. Along with its development and blooming, the red is gradually increased, and then both red and yellow were gradually faded with the wilting of petals (Figure 1A). Based on the changing style of the petal's color, we suspected that the formation of the red color is light-inducible. To verify this hypothesis, two different light treatments were carried out on the newly bloomed flowers in May 2020, natural sunlight (control group) and shading by being wrapped in a black opaque paper bag (Figure 1B). Rose flower petals at four developmental stages (named as S1, S2, S3 and S4) were observed to compare the difference of color changes between the two light treatments. At the S1 stage corresponding to the newly opened flower (Figure 1(A5)), flowers under both treatments were mainly yellow; at the S2 stage, the flower is mainly yellow with some light red (Figure 1(A7),B) under natural light, while it stayed as yellow under darkness (Figure 1); at the S3 stage, the color was deep red with fading yellow under light (Figure 1(A9),B), and flowers in the 
dark showed only fading yellow; at the S4 stage, the yellow was totally faded under both treatments, while only the flowers with light still showed red, although it was fading as well (Figure 1(A11),B). Altogether, it could be concluded that the changes in the red color are light dependent, but the accumulation and fading of yellow is not.

\section{A}

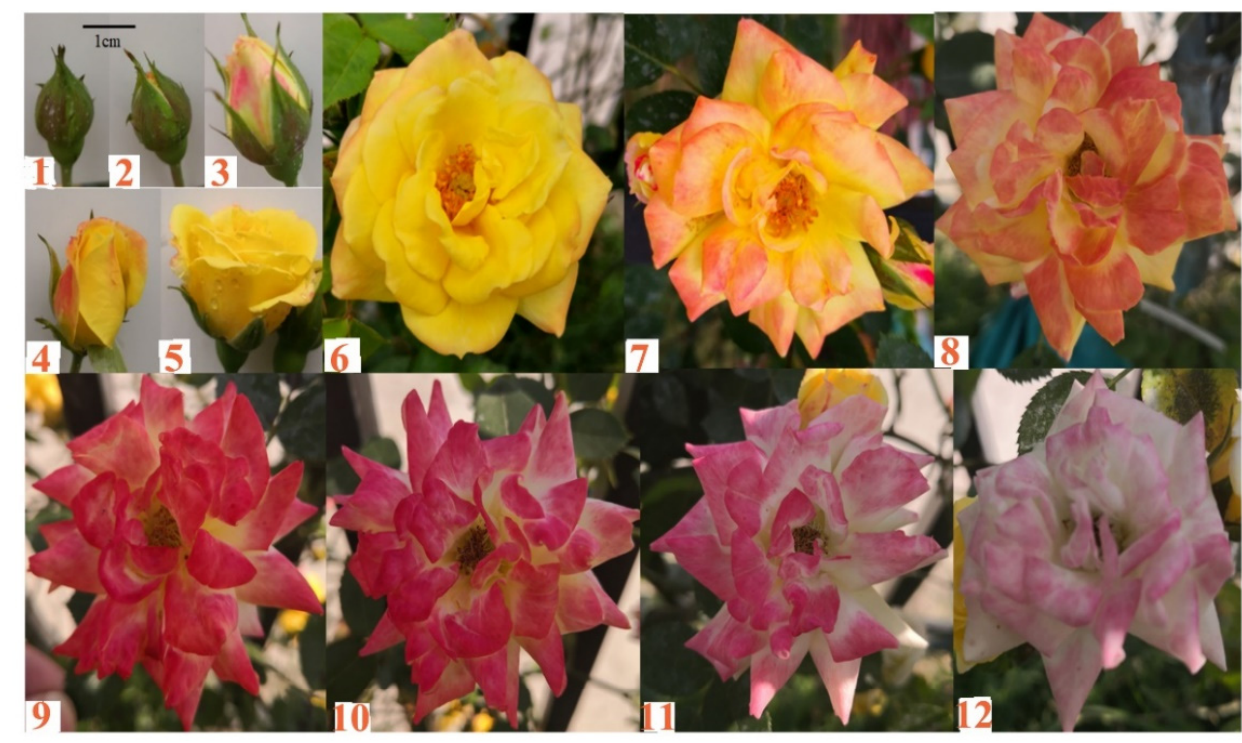

B

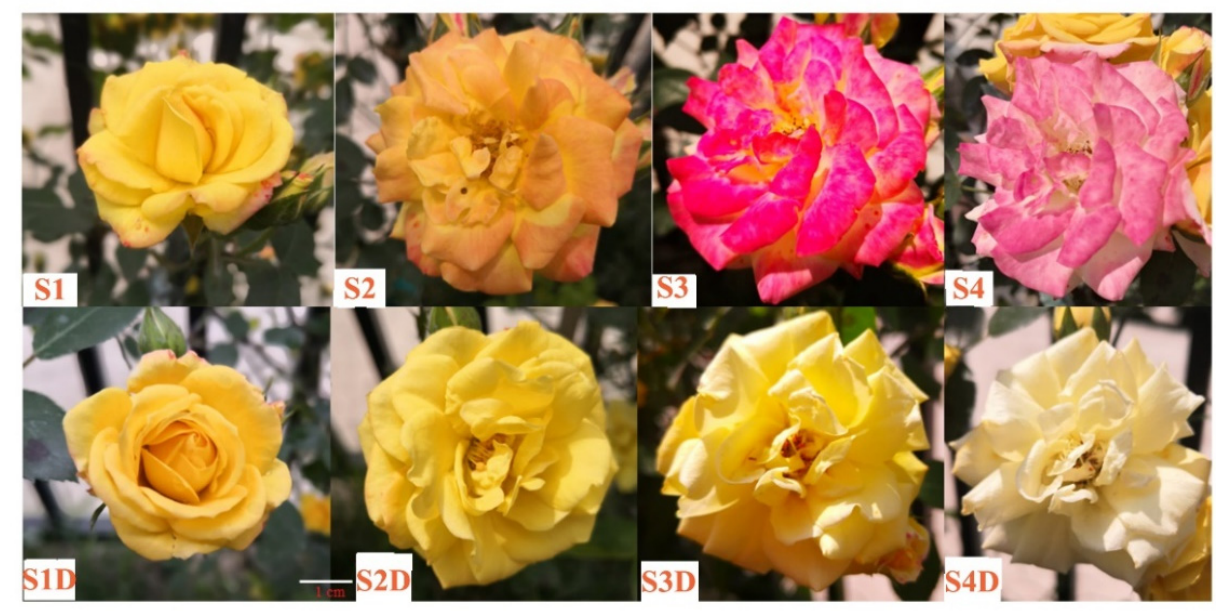

Figure 1. Changes in flower colors of the variety of 'Chen $\mathrm{Xi}^{\prime}$ rose. (A) The different developmental stages of 'Chen $\mathrm{X}$ ' rose flower from bud stage to flower blooming, and the flower color changed from yellow to red, and faded to white as it withers ((1-12) represent the order of the development stages of 'Chen $\mathrm{Xi}^{\prime}$ flower). (B) 'Chen $\mathrm{Xi}^{\prime}$ ' flowers at four blooming stages under natural sunlight (S1-S4) and shading (S1D-S4D).

\subsection{Metabolic Profiling of Rose Petals at Different Development Stages}

To explore the mechanism underlying this light-induced color change, the flavonoid metabolites of the rose petals from four different development stages and petals at the S2D stage under shading treatment (Figure 1B) were investigated by UPLC-ESI-MS/MS. A total of 176 flavonoid components were detected, including 49 flavones, 59 flavonols, 12 flavanones, 3 isoflavones, 12 anthocyanins, and 41 proanthocyanidins (Table S1), and HPLC chromatograms of these flavonoid metabolites from rose petal samples were shown in Figure S1. Principal components analysis (PCA) was carried out to assess the repeatability among three biological repeats and the variation among different samples. In the present study, two principal components PC1 and PC2 were extracted, which contributed $33.6 \%$ and $16.9 \%$, respectively. The mix represents a sample for quality control, and PCA 
shows separation of the different samples and good repeatability (Figure 2). Additionally, samples of S2D could be separated from other samples by PCA (Figure 2).

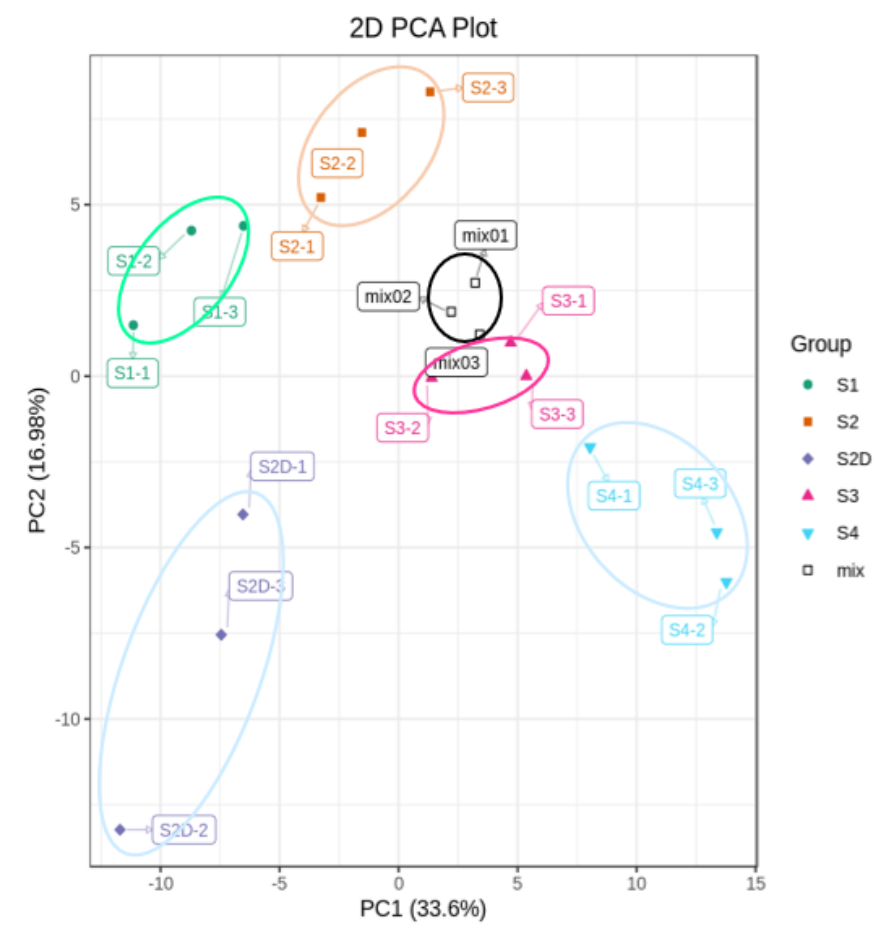

Figure 2. Principal component analysis (PCA) of flavonoid metabolites profiles analysis.

All the metabolites are illustrated by a heatmap, and six groups were classified based on the content of metabolites in different samples (Figure 3A). Metabolites in Group I, which had the highest number of compounds (52), mainly composed of flavones, flavonols and proanthocyanidins, had lower content in the petals of S1, then most of them increased along with the growth of the rose flower development, and there was no significant difference between S1 and S2D petals, but most of the metabolites were higher in S2 than S2D petals, while most metabolites in group II, including flavones, proanthocyanidins, flavanols, and flavonols, had the lowest content in the shading treatment (in S2D petals) but had the highest content in S2 petals. In group III, some metabolites exhibited no difference between $\mathrm{S} 1$ and S2 petals, then slowly increased in S3 and accumulated more in S4 petals, while some were lower in S1 petals, increased in S2 and S3, then sharply down regulated in S4 petals. Most of the metabolites in this group had higher content in petals S2D than that in S1 and S2 petals. This group also mainly contained flavones, flavonols and proanthocyanidins. Group IV had the lowest number of members, including four flavones, four flavonols, one flavanol, one anthocyanin and one proanthocyanin, almost all of them showed the highest content in S1 petals, and decreased rapidly in S2, and kept declining in S3 and S4 petals, but only several members exhibited up-regulation in S4. The majority of metabolites in the S2D of this group had slightly lower content than those in S1. In group V, flavonols and proanthocyanidins were the main members, and they kept a high content in the first two stages petals but showed a continued descent in petals of S3 and S4 petals, with most metabolites being down-regulated in S2D petals. Unlike other groups, most of the flavonoid metabolites in group VI, which included 12 flavones, 8 proanthocyanidins, 4 flavonols, 2 anthocyanins, and 1 flavanol, had the highest content in S2D, while they had a lower expression in S1, S3, and S4 flowers (Figure 3A). Specifically, eriodictyol (dihydroflavone), quercetin-3-O-(2"-acetyl)-glucosylgalactoside (flavonol), cyanidin-3-O-glucoside (kuromanin), and cyanidin-3-O-(6"-malonylglucoside) (two anthocyanins) showed a dramatically light-induced pattern, whereas two proanthocyanidins valoneoyl-glucose and procyanidin A6 showed an opposite pattern (Table 1). 
A

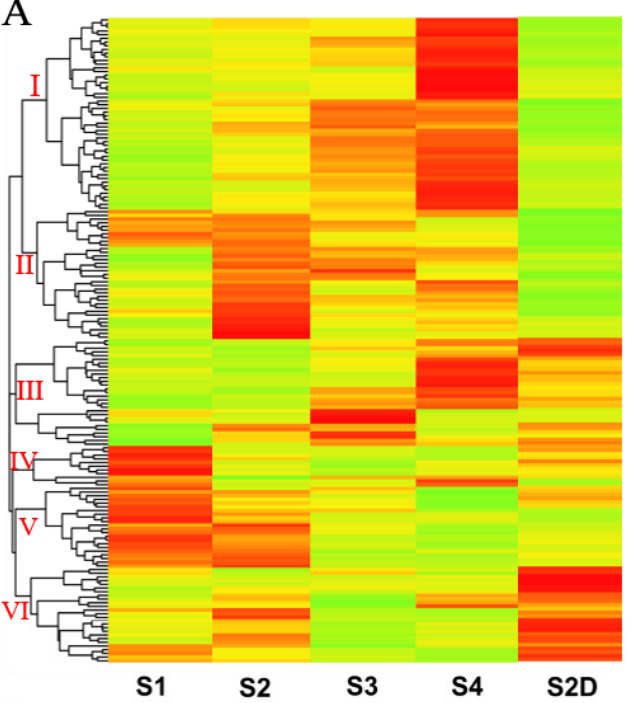

$\mathrm{C}$

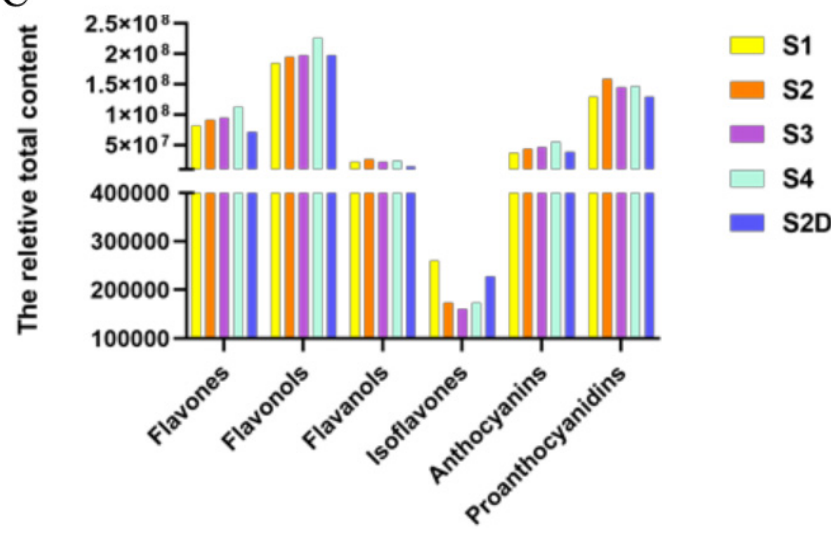

$\mathrm{B}_{\mathrm{I}(52)}$

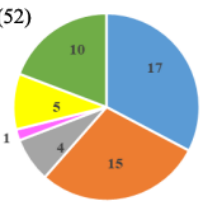

II (35)

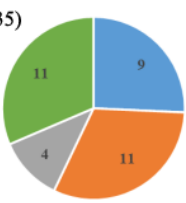

$-0.5$

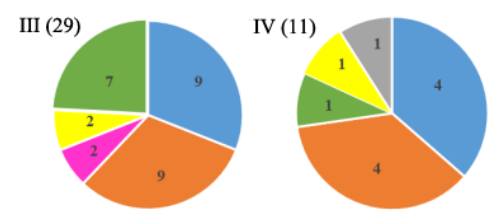

$\mathrm{V}(22)$

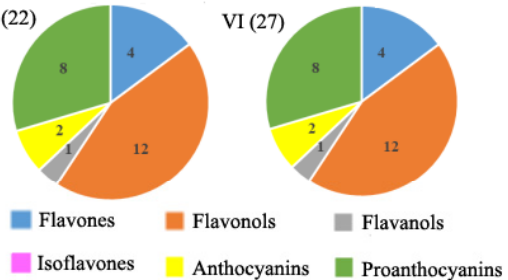

Isoflavones Anthocyanins $\quad$ Proanthocyanins

Figure 3. Flavonoid metabolites profile analysis. (A) Clustering heat map of all flavonoid metabolites, and (B) pie charts of the metabolites in each group. (C) The relative content of six types of flavonoid compounds, the content value was calculated by the peak area of each metabolite.

Moreover, the total content of six types of flavonoids in different samples was analyzed based on the peak area of each compound. As shown in Figure 3C, flavonols were the most abundant, which also had the largest number; they slightly increased in S2 and S3 rose petals and continued to rise in $\mathrm{S} 4$ petals. There was no obvious difference between S2 and S2D, but compared to S1 petals, S2D petals had a little higher content of flavonols. Proanthocyanidins were the second abundant in content, which increased in S2 petals, then slightly descended in S3 flowers and remained unchanged in S4 but showed no change in S2D compared to S1. As the second-largest number of flavonoid metabolites was detected in rose petals, flavones content also saw a sustained increase when exposed to light; however, it declined under shading light in S2D. Furthermore, the content of anthocyanin ceased to increase under shading, but continued to rise with illumination. Flavaonols exhibited no significant changes during rose flower development under both treatments. Isoflavones were the lowest in quantity and content, and they showed a declining trend from $\mathrm{S} 1$ to $\mathrm{S} 3$, then a slight rise at $\mathrm{S} 4$; however, they were expressed higher in S2D than that in S2 petals, but lower than S1 petals (Figure 3C). 
Table 1. The information of differential metabolites from five comparison groups.

\begin{tabular}{|c|c|c|c|c|c|c|c|c|c|}
\hline Index & MW (Da) & $\begin{array}{l}\text { Ionization } \\
\text { Model }\end{array}$ & Compounds & Class & S2/S1 & $\mathrm{S} 3 / \mathrm{S} 2$ & S4/S3 & S2D/S1 & S2D/S2 \\
\hline pme2960 & 272.07 & {$\left[\mathrm{M}^{+} \mathrm{H}\right]^{+}$} & Naringenin chalcone ${ }^{*}$ & Chalcones & $0.72 /$ & 0.29 & $0.70 /$ & 0.38 & $0.52 /$ \\
\hline pme1201 & 274.08 & {$\left[\mathrm{M}^{-} \mathrm{H}\right]^{-}$} & Phloretin & Chalcones & 3.29 & 0.31 & $1.44 /$ & $0.93 /$ & 0.28 \\
\hline pme0376 & 272.07 & {$\left[\mathrm{M}^{-} \mathrm{H}\right]^{-}$} & Naringenin & Dihydroflavone & $0.69 /$ & 0.29 & $0.78 /$ & 0.36 & $0.52 /$ \\
\hline Hmqp004476 & 272.07 & {$\left[\mathrm{M}^{+} \mathrm{H}\right]^{+}$} & 5,7,4'-Trihydroxydihydroflavone * & Dihydroflavone & $1.15 /$ & 0.32 & $1.06 /$ & $0.66 /$ & $0.57 /$ \\
\hline mws0064 & 288.06 & {$\left[\mathrm{M}^{-} \mathrm{H}\right]^{-}$} & Eriodictyol & Dihydroflavone & 19.10 & 0.05 & $1.75 /$ & $1.29 /$ & 0.07 \\
\hline mws1094 & 288.06 & {$\left[\mathrm{M}^{-} \mathrm{H}^{-}\right.$} & Dihydrokaempferol & Dihydroflavonol & 0.50 & 0.09 & 0.42 & 0.46 & $0.92 /$ \\
\hline mws0044 & 304.06 & {$\left[\mathrm{M}^{-} \mathrm{H}\right]^{-}$} & Dihydroquercetin (Taxifolin) & Dihydroflavonol & $1.31 /$ & $0.60 /$ & $0.63 /$ & $0.61 /$ & 0.47 \\
\hline mws1174 & 314.08 & {$\left[\mathrm{M}^{-} \mathrm{H}\right]^{-}$} & 3-O-Acetylpinobanksin & Dihydroflavonol & 7.90 & $1.06 /$ & 3.98 & 2.84 & 0.36 \\
\hline mws0040 & 254.06 & {$\left[\mathrm{M}^{+} \mathrm{H}\right]^{+}$} & Chrysin & Flavones & 6.42 & $1.35 /$ & 3.33 & 2.11 & 0.33 \\
\hline mws0051 & 284.07 & {$\left[\mathrm{M}^{+} \mathrm{H}\right]^{+}$} & Acacetin & Flavones & 2.57 & $1.49 /$ & $0.55 /$ & 2.15 & $0.84 /$ \\
\hline mws0058 & 300.06 & {$\left[\mathrm{M}^{-} \mathrm{H}\right]^{-}$} & Diosmetin & Flavones & 3.16 & $0.83 /$ & $0.51 /$ & 2.82 & $0.89 /$ \\
\hline Zmhp004065 & 344.09 & {$\left[\mathrm{M}^{+} \mathrm{H}\right]^{+}$} & $\begin{array}{c}\text { 7,8-Dihydroxy-5,6,4'- } \\
\text { trimethoxyflavone } \\
*\end{array}$ & Flavones & 6.58 & $0.99 /$ & $1.79 /$ & $0.87 /$ & 0.13 \\
\hline pmp001076 & 372.12 & {$\left[\mathrm{M}^{+} \mathrm{H}\right]^{+}$} & Isosinensetin * & Flavones & $0.88 /$ & $1.99 /$ & 0.22 & $0.62 /$ & $0.70 /$ \\
\hline mws0043 & 402.13 & {$\left[\mathrm{M}^{+} \mathrm{H}\right]^{+}$} & Nobiletin & Flavones & $0.83 /$ & 6.91 & 0.11 & $0.77 /$ & $0.93 /$ \\
\hline pmn001668 & 416.15 & {$\left[\mathrm{M}^{-} \mathrm{H}\right]^{-}$} & Apigenin-3-O-rhamnoside & Flavones & $1.56 /$ & 2.28 & $1.74 /$ & $1.40 /$ & $0.90 /$ \\
\hline Lmlp005572 & 432.11 & {$\left[\mathrm{M}^{+} \mathrm{H}\right]^{+}$} & Galangin-7-O-glucoside * & Flavones & 2.72 & $1.64 /$ & $1.45 /$ & $0.61 /$ & 0.22 \\
\hline HJN076 & 564.18 & {$\left[\mathrm{M}^{-} \mathrm{H}\right]^{-}$} & $\begin{array}{l}\text { (2R)-Pinocembrin-7-O- } \\
\text { neohesperidoside }\end{array}$ & Flavones & 5.08 & 2.97 & $1.76 /$ & 4.32 & $0.85 /$ \\
\hline Lmnp002448 & 640.16 & {$\left[\mathrm{M}^{+} \mathrm{H}\right]^{+}$} & $\begin{array}{l}5,7,3^{\prime}, 4^{\prime} \text {-Tetrahydroxy-6- } \\
\text { methoxyflavone-8-C-[glucosyl-(1- } \\
\text { 2)]-glucoside }\end{array}$ & Flavones & $1.05 /$ & 0.35 & $0.77 /$ & 0.42 & 0.40 \\
\hline Lmgn002843 & 286.05 & {$\left[\mathrm{M}^{-} \mathrm{H}\right]^{-}$} & 2'-Hydroxyisoflavone & Isoflavones & $1.97 /$ & $0.73 /$ & $1.63 /$ & $0.80 /$ & 0.41 \\
\hline mws1068 & 286.05 & {$\left[\mathrm{M}^{-} \mathrm{H}\right]^{-}$} & Kaempferol * & Flavonols & 2.03 & 0.43 & $1.55 /$ & $0.94 /$ & 0.46 \\
\hline pme3514 & 302.04 & {$\left[\mathrm{M}^{-} \mathrm{H}\right]^{-}$} & Morin * & Flavonols & 3.40 & $0.50 /$ & $1.39 /$ & $1.45 /$ & 0.43 \\
\hline pme2954 & 302.04 & {$\left[\mathrm{M}^{+} \mathrm{H}\right]^{+}$} & Quercetin & Flavonols & 3.18 & $0.52 /$ & $1.20 /$ & $1.45 /$ & 0.46 \\
\hline
\end{tabular}


Table 1. Cont

\begin{tabular}{|c|c|c|c|c|c|c|c|c|c|}
\hline Index & MW (Da) & $\begin{array}{l}\text { Ionization } \\
\text { Model }\end{array}$ & Compounds & Class & $\mathrm{S} 2 / \mathrm{S} 1$ & S3/S2 & S4/S3 & S2D/S1 & $\mathrm{S} 2 \mathrm{D} / \mathrm{S} 2$ \\
\hline mws0038 & 314.08 & {$\left[\mathrm{M}^{-} \mathrm{H}\right]^{-}$} & Kumatakenin & Flavonols & 2.57 & $1.58 /$ & $0.42 /$ & 2.69 & $1.05 /$ \\
\hline mws0917 & 330.07 & {$\left[\mathrm{M}^{-} \mathrm{H}\right]^{-}$} & 3,7-Di-O-methylquercetin & Flavonols & 5.31 & 0.46 & 2.02 & $1.40 /$ & 0.26 \\
\hline pmp000365 & 370.11 & {$\left[\mathrm{M}^{+} \mathrm{H}\right]^{+}$} & Uralenol & Flavonols & 0.48 & $0.91 /$ & $1.19 /$ & $0.63 /$ & $1.32 /$ \\
\hline mws0055 & 372.12 & {$\left[\mathrm{M}^{+} \mathrm{H}\right]^{+}$} & Tangeretin & Flavonols & $1.15 /$ & 8.18 & 0.07 & $0.83 /$ & $0.72 /$ \\
\hline Lmmn003398 & 490.11 & {$\left[\mathrm{M}^{-} \mathrm{H}\right]^{-}$} & $\begin{array}{l}\text { Kaempferol-3-O-(6"- } \\
\text { acetyl)glucoside }\end{array}$ & Flavonols & 2.03 & $1.35 /$ & $1.21 /$ & $1.56 /$ & $0.77 /$ \\
\hline pme1540 & 624.17 & {$\left[\mathrm{M}^{+} \mathrm{H}\right]^{+}$} & $\begin{array}{l}\text { Isorhamnetin-3-O- } \\
\text { neohesperidoside }\end{array}$ & Flavonols & $1.08 /$ & $0.55 /$ & 0.43 & 0.43 & 0.40 \\
\hline Hmcp001578 & 640.16 & {$\left[\mathrm{M}^{+} \mathrm{H}\right]^{+}$} & Isorhamnetin-3,7-O-diglucoside * & Flavonols & $0.78 /$ & $0.99 /$ & $0.59 /$ & 0.50 & $0.64 /$ \\
\hline Hmln001682 & 668.16 & {$\left[\mathrm{M}^{-} \mathrm{H}\right]^{-}$} & $\begin{array}{l}\text { Quercetin-3-O-(2"-acetyl)- } \\
\text { glucosylgalactoside }\end{array}$ & Flavonols & 18.83 & $0.73 /$ & $1.04 /$ & 6.54 & 0.35 \\
\hline Hmср001629 & 696.15 & {$\left[\mathrm{M}^{+} \mathrm{H}\right]^{+}$} & $\begin{array}{c}\text { Kaempferol-3-O-(6"- } \\
\text { Malonylglucoside)-7-O-Glucoside }\end{array}$ & Flavonols & 7.97 & 2.67 & $1.12 /$ & $1.93 /$ & 0.24 \\
\hline pmb0709 & 712.15 & {$\left[\mathrm{M}^{+} \mathrm{H}\right]^{+}$} & $\begin{array}{l}\text { Quercetin-7-O-malonylglucosyl- } \\
\text { glucoside } \\
*\end{array}$ & Flavonols & 4.98 & $1.39 /$ & $1.56 /$ & 2.92 & $0.59 /$ \\
\hline pmb0706 & 712.15 & {$\left[\mathrm{M}^{+} \mathrm{H}\right]^{+}$} & $\begin{array}{l}\text { Quercetin-5-O-malonylglucosyl- } \\
\text { glucoside }\end{array}$ & Flavonols & 9.37 & $1.05 /$ & $0.83 /$ & 3.50 & 0.37 \\
\hline Hmсp001757 & 756.21 & {$[\mathrm{M}+\mathrm{H}]+$} & $\begin{array}{l}\text { Quercetin-O-rhamnoside-O- } \\
\text { glucoside-O-rhamnoside }\end{array}$ & Flavonols & 3.26 & $0.89 /$ & $0.61 /$ & $1.19 /$ & 0.36 \\
\hline mws1422 & 274.08 & {$\left[\mathrm{M}^{+} \mathrm{H}\right]^{+}$} & Epiafzelechin * & Flavanols & 4.22 & $0.89 /$ & $0.74 /$ & $0.81 /$ & 0.19 \\
\hline pmn001415 & 452.11 & {$\left[\mathrm{M}^{-} \mathrm{H}\right]^{-}$} & $\begin{array}{c}\text { Catechin-(7,8-bc)-4 } 3-(3,4- \\
\text { dihydroxyphenyl)-dihydro-2- } \\
(3 \mathrm{H}) \text {-ne } \\
*\end{array}$ & Flavanols & $0.94 /$ & 2.01 & 3.10 & $1.44 /$ & $1.53 /$ \\
\hline pmn001416 & 452.11 & {$\left[\mathrm{M}^{-} \mathrm{H}\right]^{-}$} & 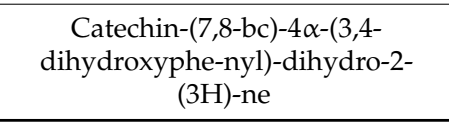 & Flavanols & $1.09 /$ & $1.70 /$ & 3.02 & $1.47 /$ & $1.35 /$ \\
\hline
\end{tabular}


Table 1. Cont.

\begin{tabular}{|c|c|c|c|c|c|c|c|c|c|}
\hline Index & MW (Da) & $\begin{array}{c}\text { Ionization } \\
\text { Model }\end{array}$ & Compounds & Class & S2/S1 & S3/S2 & $\mathrm{S} 4 / \mathrm{S} 3$ & $\mathrm{~S} 2 \mathrm{D} / \mathrm{S} 1$ & S2D/S2 \\
\hline HJN041 & 452.13 & {$\left[\mathrm{M}^{-} \mathrm{H}\right]^{-}$} & Epicatechin glucoside & Flavanols & $1.12 /$ & $0.81 /$ & $0.94 /$ & 0.46 & 0.41 \\
\hline Smlp002532 & 419.10 & {$[\mathrm{M}]^{+}$} & Cyanidin-3-O-arabinoside & Anthocyanins & 4.44 & $1.33 /$ & $1.52 /$ & $0.97 /$ & 0.22 \\
\hline pmb0550 & 449.11 & {$[\mathrm{M}]^{+}$} & $\begin{array}{c}\text { Cyanidin-3-O-glucoside } \\
\text { (Kuromanin) }\end{array}$ & Anthocyanins & 17.48 & 2.07 & $1.39 /$ & $1.36 /$ & 0.08 \\
\hline pmb0542 & 535.11 & {$[\mathrm{M}]^{+}$} & $\begin{array}{l}\text { Cyanidin-3-O-(6"- } \\
\text { Malonylglucoside) }\end{array}$ & Anthocyanins & 16.81 & 6.33 & $1.78 /$ & $1.91 /$ & 0.11 \\
\hline Lmpp003815 & 625.16 & {$[\mathrm{M}]^{+}$} & $\begin{array}{c}\text { Petunidin-3-(6"-p- } \\
\text { Coumaroylglucoside) }\end{array}$ & Anthocyanins & $0.98 /$ & $0.58 /$ & 0.40 & 0.48 & 0.49 \\
\hline Lmjp001323 & 743.20 & {$[\mathrm{M}]^{+}$} & $\begin{array}{c}\text { Cyanidin } \\
\text { 3-O-sambubioside-5-O-glucoside }\end{array}$ & Anthocyanins & $1.19 /$ & $0.83 /$ & $1.19 /$ & 0.49 & 0.41 \\
\hline mws0024 & 170.02 & {$\left[\mathrm{M}^{-} \mathrm{H}\right]^{-}$} & Gallic acid & Proanthocyanidins & 2.66 & $0.51 /$ & $1.17 /$ & $1.40 /$ & $0.53 /$ \\
\hline pmn001519 & 336.05 & {$\left[\mathrm{M}^{-} \mathrm{H}\right]^{-}$} & Galloyl Methyl gallate & Proanthocyanidins & $0.78 /$ & $0.75 /$ & $1.01 /$ & 0.50 & $0.64 /$ \\
\hline Cmsn000894 & 362.08 & {$\left[\mathrm{M}^{-} \mathrm{H}\right]^{-}$} & 7-O-Galloyl-D-sedoheptulose & Proanthocyanidins & $1.52 /$ & $1.38 /$ & $1.41 /$ & 2.01 & $1.32 /$ \\
\hline Wmhn001495 & 484.08 & {$\left[\mathrm{M}^{-} \mathrm{H}\right]^{-}$} & 1,4-Di-O-galloyl-glcose & Proanthocyanidins & $1.34 /$ & $0.97 /$ & $0.82 /$ & $0.64 /$ & 0.47 \\
\hline Cmhn000855 & 650.08 & {$\left[\mathrm{M}^{-} \mathrm{H}\right]^{-}$} & Valoneoyl-glucose & Proanthocyanidins & $1.39 /$ & $1.63 /$ & $1.39 /$ & 3.36 & 2.42 \\
\hline pme0432 & 576.13 & {$\left[\mathrm{M}^{-} \mathrm{H}\right]^{-}$} & Procyanidin A2 * & Proanthocyanidins & 2.07 & $0.70 /$ & $1.02 /$ & $0.73 /$ & 0.35 \\
\hline pme0430 & 576.13 & {$\left[\mathrm{M}^{-} \mathrm{H}\right]^{-}$} & Procyanidin A1 & Proanthocyanidins & 2.06 & $1.13 /$ & $1.56 /$ & $1.21 /$ & $0.59 /$ \\
\hline pmn001667 & 578.14 & {$\left[\mathrm{M}^{-} \mathrm{H}\right]^{-}$} & Procyanidin B4* & Proanthocyanidins & $1.03 /$ & $1.07 /$ & 2.11 & $0.60 /$ & $0.58 /$ \\
\hline HJN074 & 592.16 & {$\left[\mathrm{M}^{-} \mathrm{H}\right]^{-}$} & Procyanidin A6 & Proanthocyanidins & $0.66 /$ & $1.50 /$ & $0.86 /$ & 1.37 & 2.08 \\
\hline
\end{tabular}




\subsection{Differential Metabolite Screening, Functional Annotation, and Enrichment Analysis}

Differential flavonoid metabolites of each comparison group were screened by combing fold change $(\geq 2$ or $\leq 0.5)$ and variable importance in project (VIP, $\geq 1)$ of orthogonal signal correction and partial least squares-discriminant analysis (OPLS-DA). In total, 56 significantly differential metabolites (SDM) were screened from all the five comparison groups, including S1 VS S2, S2 VS S3, S3 VS S4, S1 VS S2D and S2 VS S2D, (Table 1). There were 30 SDM in S1 VS S2 group (28 up-regulated, 2 down-regulated), 18 between S2 and S3 (8 up-regulated, 10 down-regulated), 13 in S3 VS S4 group (7 up-regulated, 6 downregulated), 23 between S1 and S2D (12 up-regulated, 11 down-regulated), and 32 between S2 and S2D (2 up-regulated, 30 down-regulated), (Figure 4A-E). Venn diagram showed the intersection of each comparison group. However, no common differential metabolites were observed among these five comparison groups with only two metabolites showing a continued change during rose development without covering, and each comparison group had its unique differential metabolites (Figure 4F).
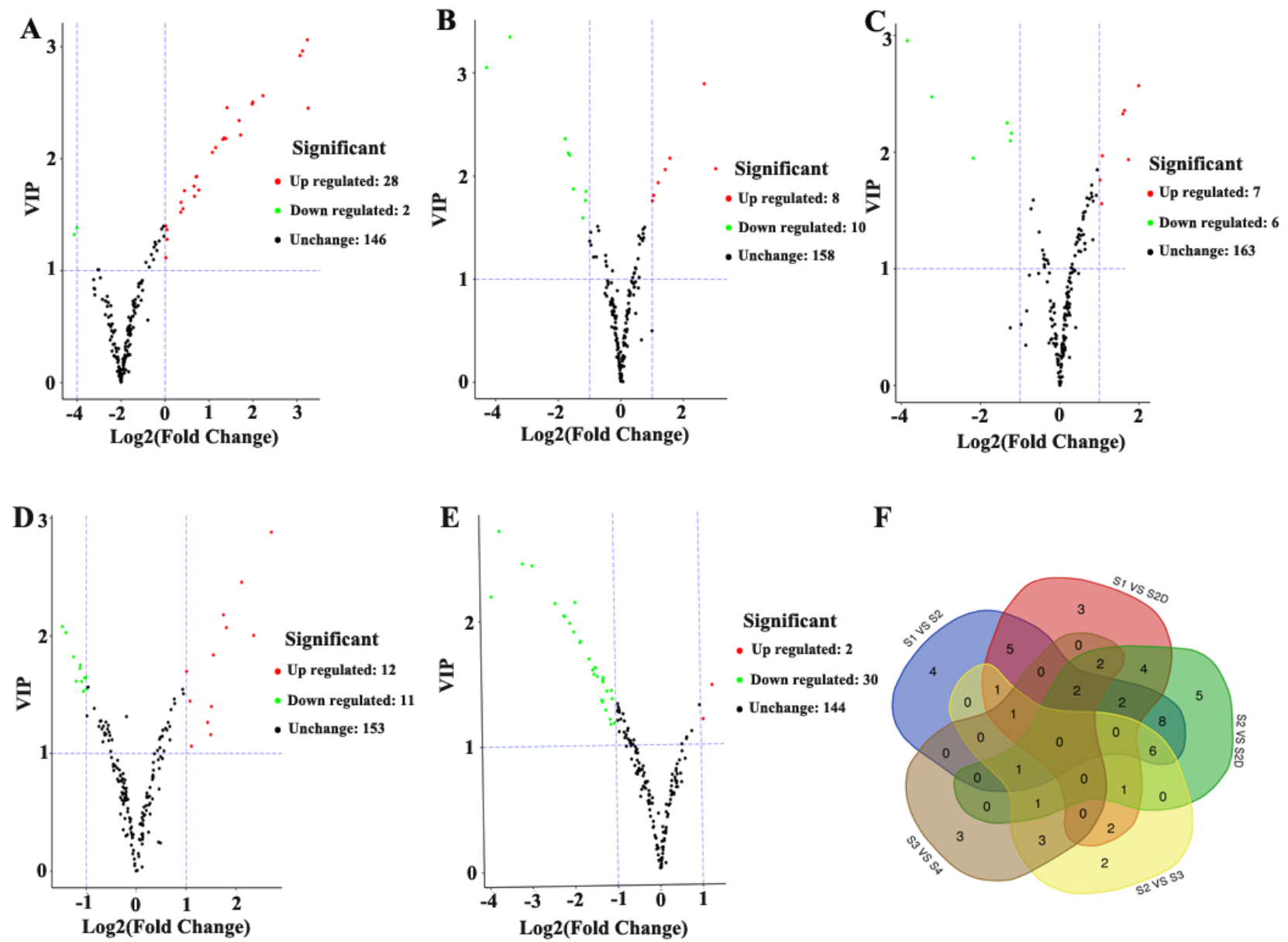

$\mathbf{F}$

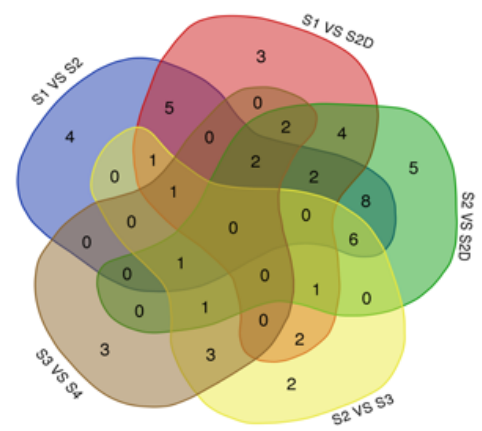

Figure 4. Differential flavonoid metabolites screening results for each comparison group by volcano plots ((A), S1 versus S2; (B), S2 versus S3; (C), S3 versus S4; (D), S1 versus S1D and (E), S2 versus S2D), and Venn diagram of these five comparison groups $(\mathbf{F})$.

After annotation by the Kyoto Encyclopedia of Genes and Genomes (KEGG) database, the differential flavonoid metabolites of each comparison group were mainly enriched in pathways including isoflavones, flavonoid, flavone and flavonol, phenylpropanoids, secondary metabolites, anthocyanin, and metabolic pathways (Figure 5). 


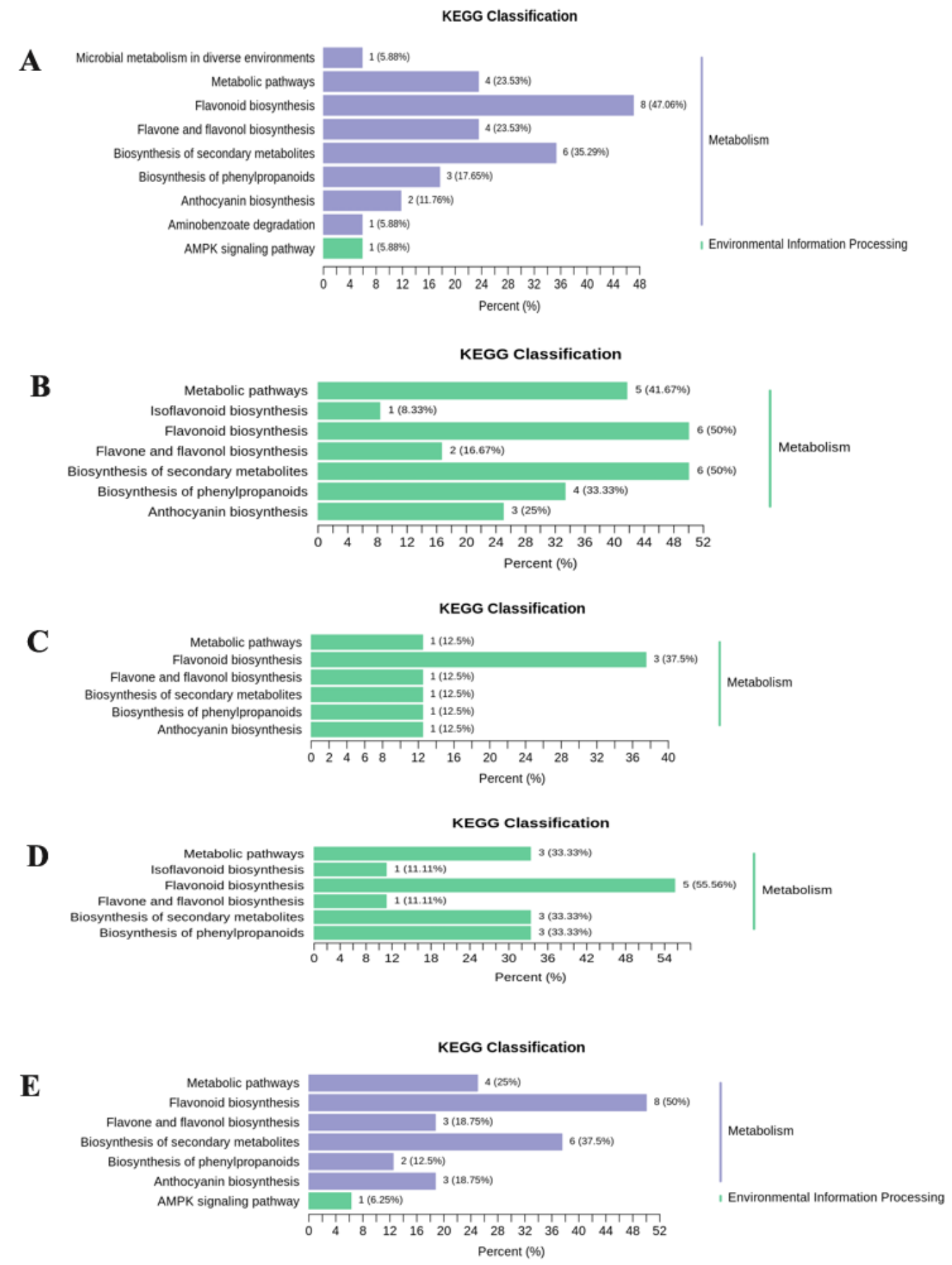

Figure 5. The differential flavonoid metabolite KEGG classification of each comparison group, (A) S1 versus S2; (B) S2 versus S3; (C) S3 versus S4; (D) S1 versus S1D; and (E) S2 versus S2D.

\subsection{The Differential Metabolites in Developing Flowers Enriched in Flavonoid Pathway}

The changing trends of these differential flavonoid metabolites (DFM) in developmental rose flowers were demonstrated by a sequence chart based on their relative content (Figure 6). For DFM in four developmental stages flowers of 'Chen $\mathrm{Xi}^{\prime}$ under natural conditions, cluster 1 contained the greatest number of DFM (19), which had the highest content in S1 petals, but continued to decline with flower development. There were five flavones, four flavonols, two flavanols, one isoflavones, three anthocyanins, and four proanthocyanidins in this cluster (Figure 5A). In cluster 2, DFM, including five flavonols, four flavones, one flavanol, one anthocyanins, and two proanthocyanidins, had the lowest expression in S1 petals, then rapidly increased in S2 petals and decreased in S3 petals, a little up-regulated 
in S4 petals. However, most of the DFM in cluster 3 were up-regulated constantly along with rose flower development, and five flavones, four flavonols, one flavanol, and two proanthocyanidins belonged to this cluster. Unlike the other three clusters, DFM in cluster 4 had the lowest content in S1 petals, then sharply rose in S2 petals, and maitained in S3 petals, declined S4 petals (Figure 6A).

A
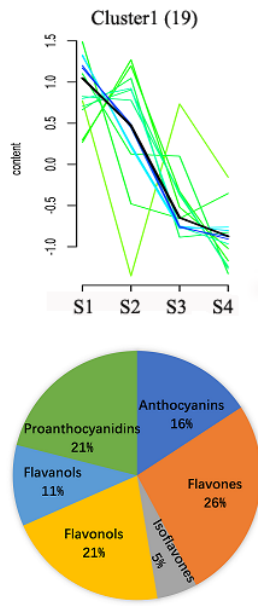

B
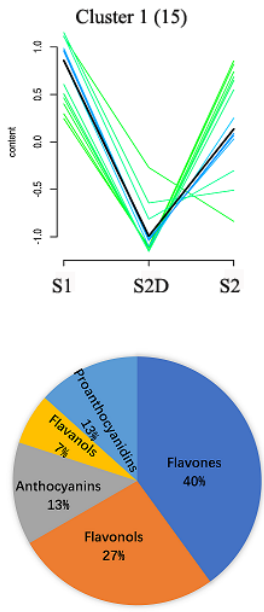
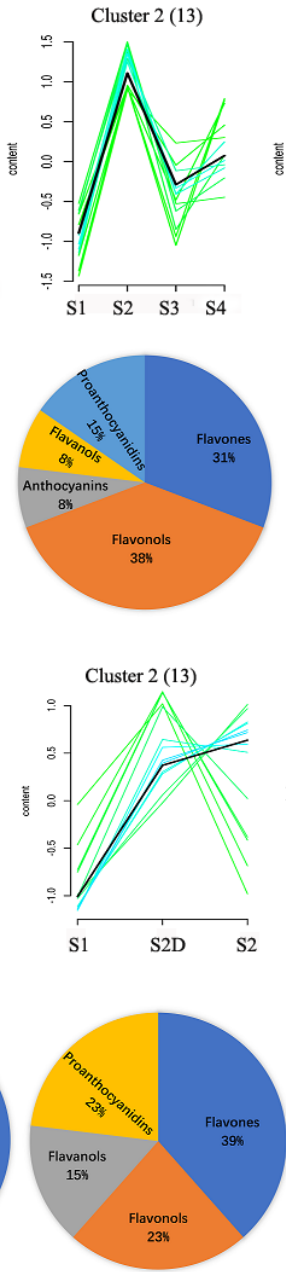
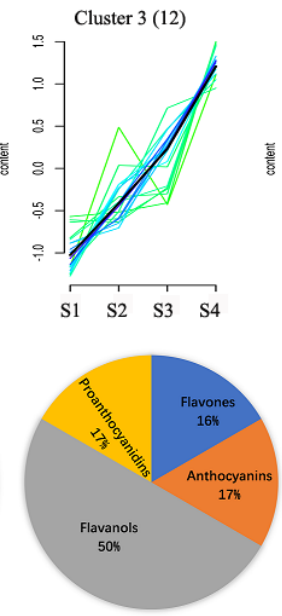
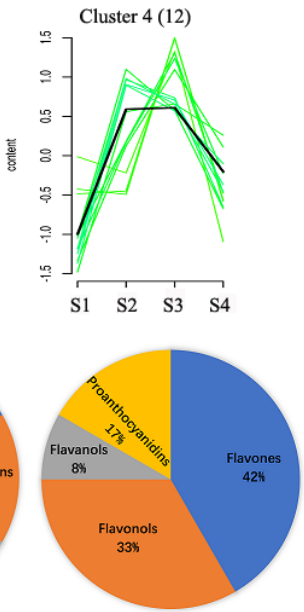

Figure 6. Sequence chart of differential metabolites in developing rose flowers and the ratio of metabolic groups for each cluster depicted above. (A) Rose flowers from S1 to S4 under natural light condition and (B) rose flowers from S1, S2D and S2.

Moreover, petals under the shading treatment in stage 2 (S2D) had different changes compared with petals under the natural light condition in S2, which were classified into 3 clusters (Figure 5B). DFM in cluster 1 were down-regulated in S2D petals, but upregulated in S2 petals. This cluster contained 15 members, and flavones took up the greatest percentage $(40 \%)$, followed by flavonols (27\%) (Figure 5B). In cluster 2, DFM both in S2 and S2D petals were up-regulated compared to S1 petals, but DFM had higher content in S2 petals than in S2D petals. Flavones were the most abundant in cluster $2(39 \%)$, then followed by flavonols and proanthocyanidins (23\%). In addition, cluster 3 contained the greatest number of DFM (28), which showed no significant change between S1 and S2D petals, but sharply accumulated in S2 petals, and six type of flavonoids were contained in this cluster; among them, flavonols were the most abundant (Figure 6B).

All the DFM were enriched in the flavonoid biosynthesis pathway (Figure 7). There were two chalcones; one was the most abundant in S1 flowers, and decreased in the following development stages, and it was expressed more in S2 petals than in S2D petals. The other one increased sharply from S1 to S2 but declined quickly in flowers S3 and S4. 
Moreover, compared to the S1 flower, it exhibited no change in the S2 flower without illumination. Only one type of isoflavone showed differences among samples, and it showed a strange change trend, which had lower content in S1 flowers, then rose in S2 flowers, but decreased in S3 flowers and increased again and reached the highest content in S4 flowers, while it had lower content in S2D flowers. Among the 11 flavanones, the content of five members increased along with flower development, while they showed no change between S1 and S2D flowers. Only one had higher content in S1 flower, two of them showed higher content in S2D flower, and two had the highest content in S3 flowers. Six dihydroflavonols showed a different expression trend; five of them were more abundant in S1 and S2 flowers, and the remaining one was only expressed highly in S4 flowers and had lower content in other stages' flowers. The number of flavonol and derivatives is the largest (16); three of them had a higher content in S1 flowers and most members had a higher content in the flowers of S2, S3, and S4. However, all the members showed lower expression levels in S2D. Four flavanols displayed differential expression levels among different development of flowers, two of them had lower content in the first three stages, but quickly increased in S4 flowers; one was expressed the lowest only in S2D, and one had a lower content in S1 and S2D flowers, then ascended quickly in S2 flowers and slowly decreased in S3 and S4 flowers. The last one was expressed higher in S1 and S2 flower, and a slight reduction in S3 and S4 flowers. Ten proanthocyanidins were detected with differentially expressed levels among samples, and most of them had higher content in the flowers of S2, S3, and S4, several of them expressed more in S1 flowers or S2 flowers. There were six anthocyanins that showed different expression patterns during rose flower development. Three of them displayed a gradual increase along with flower development, while the other three had higher expression levels in S2 flowers. However, all of them had lower expression in S2 flowers without illumination (Figure 7).

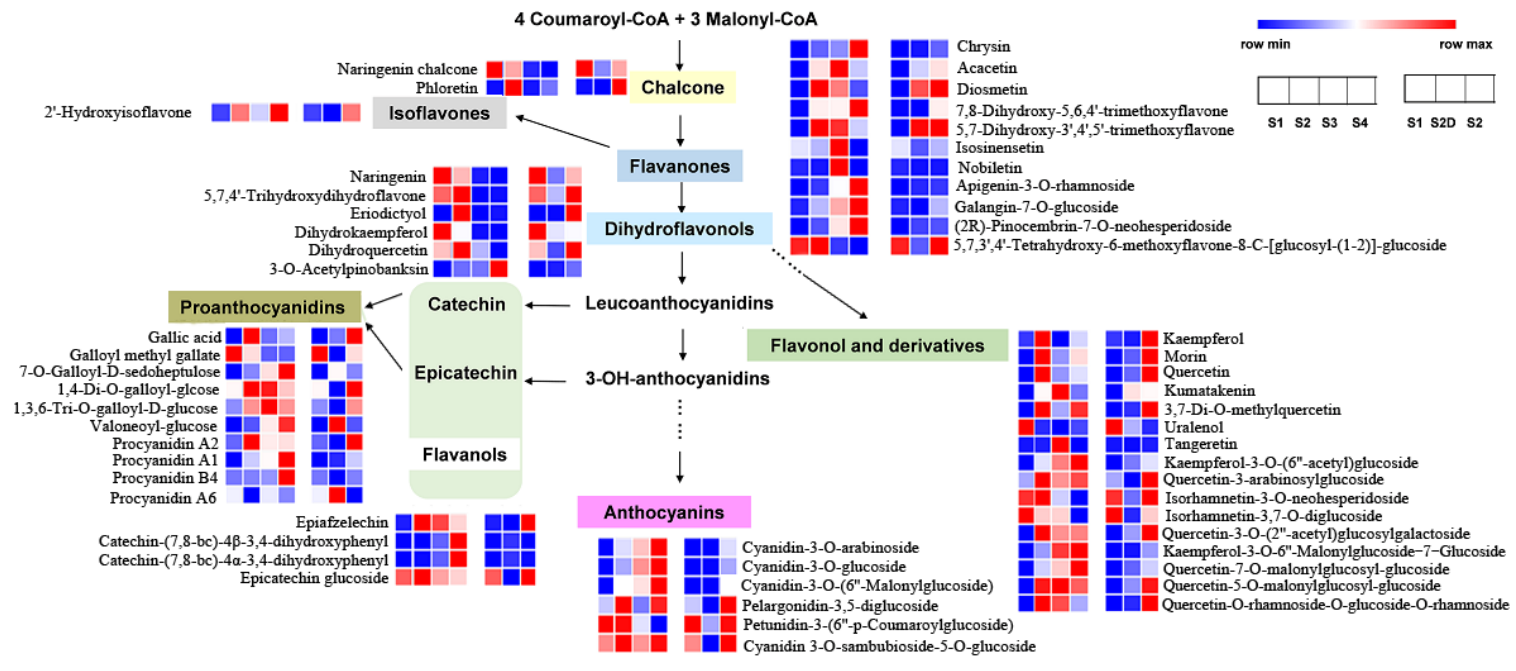

Figure 7. Differential metabolite enrichment in flavonoid biosynthesis and their accumulation profiles in developing rose flowers by heatmap.

Overall, during the petal color changing progress, most of the flavonoid metabolites were accumulated from S1 to S2; only two flavonols exhibited a significant decrease. Eight compounds including three flavones, two flavonols, one flavanol, and two anthocyanins were decreased from S2 to S3, while eight flavones and one anthocyanin significantly increased from S2 to S3. From S3 to S4, seven compounds (one flavone, two flavonols, two flavanols, one anthocyanin, and one proanthocyanidin) and six compounds (three flavonols, two flavones, and one anthocyanin) were significantly increased and decreased, respectively (Table 1 ).

When treated by shading, 11 flavonoid compounds (5 flavonols, 4 flavones and 2 proanthocyaidins) significantly increased from S1 to S2D petals, but almost all of them 
decreased in the S2D vs. S2 comparison group (Table 1). Except for two proanthocyanidns (valoneoyl-glucose and procyanidin A6), whose content was obviously more abundant in S2D petals compared with that in S2 and S1 and S2, while the other 30 compounds (5 flavones, 1 isoflavone, 13 flavonols, 2 flavanols, 6 anthocyanins and 3 proanthocyanidins) were decreased greater in S2D petals than in S2 petals.

Since the red color was determined by anthocyanins, based on the accumulation profiles of these six anthocyanins, although all of their content sharply declined in shading petals compared with $\mathrm{S} 2$ petals, only three cyanidin derivatives (anidin-3-O-arabinoside, cyanidin-3-O-glucoside and cyanidin-3-O-(6"-Malonylglucoside)) exhibited sustained rapid accumulation during flower blooming, and keep nearly the same content in S1 and S2D (Table 1, Figure 7), which was consistent with the color. Thus, we speculated that these three anthocyanins mainly contributed to the red color of petals.

\section{Discussion}

The 'Chen $\mathrm{Xi}^{\prime}$ rose, whose petal colors can be changed during the flowering process, has highly ornamental value. Previous studies reported that the yellow color of the rose is determined by carotenoids, while the red color is mainly caused by anthocyanins $[3,10]$. Furthermore, rose petals have been widely used as traditional medicinal therapy, food additives, as well as for cosmetics due to these abundant bioactive compounds $[4,11,16]$. Here, we detected the flavonoid metabolites during different developmental stages of this bicolored rose flower, with one stage (S2) subjected to shading treatment.

\subsection{Abundant Flavonoid Metabolites Accumulated in Rose Flower Petals}

In total, 176 flavonoid metabolites were identified, containing 49 flavones, 59 flavonols, 12 flavanones, 3 isoflavones, 12 anthocyanins, and 41 proanthocyanidins in this bicolored rose flower (Table S1). Among them, many metabolites were found for the first time. Previous studies identified four anthocyanin compounds, including cyanidin 3-O-glucoside, pelargonidin 3-glucoside, cyanidin 3,5-di-O-glucoside and pelargonidin 3,5-di-O-glucoside [10-12], which belongs to single or di-glycoside derivatives of cyanidin and pelargonidin. However, in this study, only two anthocyanins were the same as before (cyanidin-3-O-glucoside and pelargonidin-3,5-diglucoside), and five other cyanidins and one petunidin were glycosylated by other types of glycosides, including arabinoside, malonylglucoside, sambubioside, and coumaroylglucoside with single or diosaccharide. Other kinds of anthocyanin compounds with different glucosides were detected, such as delphinidin-3-O-glucoside, malvidin-3-O-malonylglucoside and petunidin-3-(6"-p-Coumaroylglucoside) (Table S1). Only 20 flavones, including 16 kaempferol and derivatives and 3 quercetins and derivatives as well as one flavan-3-ol derivative, were identified before [10]; however, 59 flavonols and other flavonoid compounds were detected (Table S1) which border the flavonoid metabolites in rose flowers. These results showed that rose flowers are rich in flavonoid metabolites, with their content being high during the blooming stages. Flavonoids, especially the high content of flavonols in rose flowers, were reported to have several bioactivities, such as antioxidant, anti-inflammatory, and anticancer roles [17-19]. Moreover, yellow rose flowers contain abundant carotenoids [10], which play vital roles in human and animal nutrition and in reducing the risk of vitamin A deficiency, cancer, and cardiovascular diseases [20,21]. Therefore, 'Chen $\mathrm{Xi}^{\prime}$ flowers may have a higher medicine value for being rich in both flavonoids and carotenoids.

\subsection{Light Affects Certain Flavonoid, Especially Anthocyanin, Biosynthesis of Rose Flowers}

Light is important for plant growth and photomorphogenesis, and it affects the accumulation of pigment in plant organs, including fruit skin, such as pear [13,22], grape [15], strawberry [23], and apple [14] and flower petals, such as Lily [24], Lisianthus [25], Gerbera [26]. These organs' color is due to anthocyanin pigment whose biosynthesis is dependent on light induction. 
In this study, the rose flower bud was yellow, but the part which was exposed to sunlight turned red, and the petals become redder along with the blooming stage after it totally opens, shadowing the yellow color. However, when the flower was grown under the shade, the flower remained yellow, which was consistent with anthocyanin accumulation. Therefore, it can be inferred that light promotes anthocyanin accumulation in rose petals, but cannot affect carotenoids biosynthesis. Furthermore, the light also affects other flavonoid compounds' biosynthesis, since most of them increased with the rose flower development under sunlight. It is a fantastic phenomenon, and we will identify this hypothesis from gene expression level in the next step, and study how light induces anthocyanin biosynthesis. It will be an interesting subject worth exploring further.

\section{Materials and Methods}

\subsection{Plant Materials and Sample Collection}

Roses were planted in the rose display area of Hubei University $\left(30^{\circ} 56^{\prime} \mathrm{N}, 114^{\circ} 32^{\prime} \mathrm{E}\right)$. The flower development from little bud to color fading was detected in April 2020 (Figure 1A). After the flower was fully opened, two different light treatments were carried out: natural sunlight (control group) and shading (Figure 1B). The shading treatment was carried out by wrapping the flower with a black opaque paper bag. Rose flower petals at four development stages (S1-S4), which were defined in Section 2.1, and one stage of flowers (corresponding to S2 flower) under light shading (Figure 1B and Figure S1(S2D)) were collected as samples. Three biological replicates were performed for each sample. The samples were then frozen in liquid nitrogen immediately and stored at $-80^{\circ} \mathrm{C}$ for further metabolite analysis.

\subsection{Sample Preparation and Extraction}

The freeze-dried rose petals were crushed by a mixer mill (MM 400, Retsch, Laichi, Germany) with a zirconia bead at $30 \mathrm{~Hz}$ for $1.5 \mathrm{~min}$. About $100 \mathrm{mg}$ of powder was weighted and extracted at $4{ }^{\circ} \mathrm{C}$ with $1.0 \mathrm{~mL} 70 \%$ aqueous methanol overnight. After being centrifuged at 10,000 $\mathrm{g}$ for $10 \mathrm{~min}$, the extracts were absorbed (CNWBOND Carbon-GCBSPE Cartridge, $250 \mathrm{mg}, 3 \mathrm{~mL}$; ANPEL, Shanghai, China, www.anpel.com.cn/cnw, accessed on 23 September 2020) and filtrated using $0.22 \mu \mathrm{m}$ filter (SCAA-104, ANPEL, Shanghai, China, http:/ / www.anpel.com.cn/, accessed on 23 September 2020) LC-MS analysis.

\subsection{HPLC Conditions}

HPLC analysis was carried according to the method described by Wang et al. [27]. A $5 \mu \mathrm{L}$ volume of each sample extract was analyzed using an LC-ESI-MS/MS system (HPLC, Shim-pack UFLC SHIMADZU CBM30A system, www.shimadzu.com.cn/, accessed on 23 September 2020; MS, Applied Biosystems 4500 Q TRAP, www.appliedbiosystems.com. $\mathrm{cn} /$, accessed on 23 September 2020). The analytical conditions were set as follows, chromatographic column, Waters ACQUITY UPLC HSS T3 C18 $(1.8 \mu \mathrm{m}, 2.1 \mathrm{~mm} \times 100 \mathrm{~mm})$; solvent system, water (containing $0.04 \%$ acetic acid): acetonitrile (containing $0.04 \%$ acetic acid); the gradient program, 100:0 V/V at $0 \mathrm{~min}, 5: 95 \mathrm{~V} / \mathrm{V}$ at $11.0 \mathrm{~min}, 5: 95 \mathrm{~V} / \mathrm{V}$ at $12.0 \mathrm{~min}, 95: 5 \mathrm{~V} / \mathrm{V}$ at $12.1 \mathrm{~min}, 95: 5 \mathrm{~V} / \mathrm{V}$ at $15.0 \mathrm{~min}$; flow rate, $0.40 \mathrm{~mL} / \mathrm{min}$; temperature, $40{ }^{\circ} \mathrm{C}$. The effluent was alternatively connected to an ESI-triple quadrupole-linear ion trap (QTRAP)-MS.

\subsection{ESI-Q TRAP-MS/MS}

Mass spectrometry was conducted following the protocol described previously $[28,29]$. The API 4500 triple quadrupole-linear ion trap mass spectrometer (Q TRAP) LC/MS/MS System, coupled with an ESI Turbo Ion-Spray interface was applied to generate the LIT and triple quadrupole (QQQ) scans, which is operating in a positive ion mode under the control of Analyst 1.6.3 software (AB Sciex). The operation parameters of the ESI source were set as below: ion source, turbo spray; source temperature, $550^{\circ} \mathrm{C}$; ion spray voltage (IS), $5500 \mathrm{~V}$; ion source gas I (GSI), gas II (GSII), curtain gas (CUR) was set at 55, 60, and 25.0 psi, 
respectively; the collision gas (CAD) was high. Instrument tuning and mass calibration were performed with 10 and $100 \mu \mathrm{mol} / \mathrm{L}$ polypropylene glycol solutions in QQQ and LIT mode, respectively. QQQ scans were acquired as MRM experiments with collision gas (nitrogen) set to 5 psi. Decluttering potential (DP) and collision energy (CE) for individual MRM transitions were performed with further DP and CE optimization. Based on the metabolites eluted within each period, we monitored a specific set of MRM transitions.

\subsection{Qualitative and Quantitative Analysis of Metabolites from Rose Flowers}

A widely targeted metabolome method constructed by Metware Biotechnology Co., Ltd. (Wuhan, China) (http:/ / www.metware.cn/, accessed on 23 September 2020) was applied to qualitatively analyze the flavonoid metabolites based on both the public available and self-built database MWDB. Additionally, a multiple reaction monitoring (MRM) method was applied for the quantification analysis of metabolites, which, using the peak area of each chromatographic peak, represents the relative content of the corresponding substance, and the information of metabolite retention time and peak pattern was used to calibrate the peaks of mass spectra detected for each compound in different samples.

\subsection{Statistical Analysis}

Three biological replicates were performed in the present study. Hierarchical clustering analysis and principal component analysis (PCA) were carried out by R software (http:/ / www.r-project.org/, accessed on 23 September 2020). The analysis on the content of different kinds of flavonoid was performed using Microsoft Office Excel 2016 (Microsoft Corporation., Redmond, WA, USA).

\section{Conclusions}

Taken together, our study revealed that light induces pigmentation changes in the petals of the 'Chen $\mathrm{Xi}$ ' rose variety. When exposed to light, the 'Chen $\mathrm{Xi}^{\prime}$ ' rose flower color changed from yellow to red, but the flower remains yellow under darkness. Flavonoid metabolites were detected during the 'Chen $\mathrm{Xi}^{\mathrm{i}}$ flower color changing process, among a total of 176 flavonoid compounds, 56 compounds showed different accumulation profiles, including 19 flavonols, 16 flavones, 1 isoflavone, 4 flavanols, 6 anthocyanins, and 10 proanthocyanidins. Some compounds increased, while some decreased accumulation during flower development; however, the total content of most of them were increased, especially flavonols, flavones and anthocyanins. Anthocyanins were the main compounds, especially three cyanidin derivatives that determined the flower's red color. These results lay a foundation for further study on the mechanism of light-induced anthocyanin biosynthesis and facilitate to breed new rose cultivar colors.

Supplementary Materials: The following is available online at https://www.mdpi.com/article/10 $.3390 /$ plants10102065/s1, Table S1: The information of the flavonoid metabolites identified in rose petals. Figure S1: The HPLC chromatograms of flavonoid metabolites from rose petal samples.

Author Contributions: Conceptualization, P.Y. and J.D.; methodology, M.S. and J.D.; validation, M.S., J.D. and R.N.D.; formal analysis, Z.H.; investigation, Z.H.; resources, M.S.; data curation, J.D.; writing-original draft preparation, M.S. and J.D.; writing-review and editing, R.N.D. and P.Y.; visualization, J.D.; supervision, P.Y.; project administration, Z.H.; funding acquisition, J.D., R.N.D. and Z.H. All authors have read and agreed to the published version of the manuscript.

Funding: This research was funded by the 2019 innovative research posts for Hubei provincial post doctors awarded (080-090469; 080-090463) to Deng J., and Rebecca ND., respectively, and Nature Science Foundation of Hubei Province (2020CFB257) to Hu Z.R.

Institutional Review Board Statement: Not applicable.

Informed Consent Statement: Not applicable.

Data Availability Statement: The data presented in this study are available in supplementary material. 
Acknowledgments: The authors are grateful to Zhiqi Yang from The No. 1 primary school of Shuiguohu Wuchang, Wuhan for her help in sample treatments and harvesting.

Conflicts of Interest: The authors declare no conflict of interest.

\section{References}

1. Guoliang, W. History of roses in cultivation I Ancient Chinese Roses. Encycl. Rose Sci. 2003, 387-395. Available online: https:/ / coek.info/queue/pdf-history-of-roses-in-cultivation-ancient-chinese-roses-.html (accessed on 23 September 2021).

2. Raymond, O.; Gouzy, J.; Just, J.; Badouin, H.; Verdenaud, M.; Lemainque, A.; Vergne, P.; Moja, S.; Choisne, N.; Pont, C.; et al. The Rosa genome provides new insights into the domestication of modern roses. Nat. Genet. 2018, 50, 772-777. [CrossRef]

3. Wan, H.H.; Yu, C.; Han, Y.; Guo, X.; Ahmad, S.; Tang, A.; Wang, J.; Cheng, T.; Pan, H.; Zhang, Q. Flavonols and carotenoids in yellow petals of rose cultivar (rosa 'sun city'): A possible rich source of bioactive compounds. J. Agric. Food Chem. 2018, 66, 4171-4181. [CrossRef]

4. Guimarães, R.; Barros, L.; Carvalho, A.M.; Ferreira, I.C.F. Studies on chemical constituents and bioactivity of Rosa micrantha: An alternative antioxidants source for food, pharmaceutical, or cosmetic applications. J. Agric. Food Chem. 2010, 58, 6277-6284. [CrossRef] [PubMed]

5. Héthelyi, B.; Szarka, S.; Lemberkovics, É.; Szke, É. SPME-GC/MS identification of aroma compounds in rose flowers. Acta Agron. Hung. 2010, 58, 283-287. [CrossRef]

6. Ibrahim, M.; Du, X.; Agarwal, M.; Hardy, G.; Abdulhussein, M.; Ren, Y. Influence of benzyladenine on metabolic changes in different rose tissues. Plants 2018, 7, 95. [CrossRef] [PubMed]

7. Prata, G.G.; Souza, K.O.D.; Lopes, M.M.D.; Aragão, F.A.S.D.; Oliveira, L.D.S.; Alves, R.E.; Silva, S. Nutritional characterization, bioactive compounds and antioxidant activity of brazilian roses (Rosa spp.). J. Agric. Sci. Technol. 2017, 19, $929-941$.

8. Nanjaraj, U.A.N.; Hu, Y.; Li, P.; Michael, Y.; Chen, Y.; Zhang, Y. Cloning and Expression of a Nonribosomal Peptide Synthetase to Generate Blue Rose. ACS Synth. Biol. 2019, 8, 1698-1704. [CrossRef]

9. Schmitzer, V.; Veberic, R.; Osterc, G.; Strampar, F. Color and phenolic content changes during flower development in groundcover rose. J. Am. Soc. Hortic. Sci. 2010, 135, 195-202. [CrossRef]

10. Wan, H.; Chao, Y.; Han, Y.; Guo, X.; Luo, L.; Pan, H.; Zheng, T.; Wang, J.; Cheng, T.; Zhang, Q. Determination of flavonoids and carotenoids and their contributions to various colors of rose cultivars (Rosa spp.). Front. Plant Sci. 2019, 10, 123. [CrossRef]

11. Lee, J.H.; Lee, H.; Choung, M. Anthocyanin compositions and biological activities from the red petals of Korean edible rose (Rosa hybrida cv. Noblered). Food Chem. 2011, 129, 272-278. [CrossRef] [PubMed]

12. Huang, P.; Lin, F.; Li, B.; Zheng, Y. Hybrid-Transcriptome sequencing and associated metabolite analysis reveal putative genes involved in flower color difference in rose mutants. Plants 2019, 8, 267. [CrossRef] [PubMed]

13. Bai, S.; Tao, R.; Tang, Y.; Yin, L.; Ma, Y.; Ni, J.; Yan, X.; Yang, Q.; Wu, Z.; Zeng, Y.; et al. BBX16, a B-box protein, positively regulates light-induced anthocyanin accumulation by activating MYB10 in red pear. Plant Biotechnol. J. 2019, 17, 1985-1997. [CrossRef] [PubMed]

14. Fang, H.; Dong, Y.; Yue, X.; Hu, J.; Jiang, S.; Xu, H.; Wang, Y.; Su, M.; Zhang, J.; Zhang, Z.; et al. The B-box zinc finger protein MdBBX20 integrates anthocyanin accumulation in response to ultraviolet radiation and low temperature. Plant Cell Environ. 2019, 42, 2090-2104. [CrossRef] [PubMed]

15. Sun, L.; Li, S.; Tang, X.; Fang, X.; Zhang, Y.; Jiang, J.; Liu, J.; Liu, C. Transcriptome analysis reveal the putative genes involved in light-induced anthocyanin accumulation in grape 'Red Globe' ( $V$. vinifera L.). Gene 2020, 728, 144284. [CrossRef]

16. Sarangowa, O.; Kanazawa, T.; Nishizawa, M.; Myoda, T.; Bai, C.; Yamagishi, T. Flavonol glycosides in the petal of Rosa species as chemotaxonomic markers. Phytochemistry 2014, 107, 10761-10768. [CrossRef]

17. Kim, H.P.; Son, K.H.; Chang, H.W.; Kang, S.S. Anti-inflammatory plant flavonoids and cellular action mechanisms. J. Pharmacol. Sci. 2004, 96, 229-245. [CrossRef]

18. Ren, W.; Qiao, Z.; Wang, H.; Zhu, L.; Zhang, L. Flavonoids: Promising anticancer agents. Med. Res. Rev. 2003, $23,519-534$. [CrossRef] [PubMed]

19. Zhang, S.; Lu, B.; Han, X.; Xu, L.; Qi, Y.; Yin, L.; Xu, Y.; Zhao, Y.; Liu, K.; Peng, J. Protection of the flavonoid fraction from Rosa laevigata Michx fruit against carbon tetrachloride-induced acute liver injury in mice. Food Chem. Toxicol. 2019, 132, 110695. [CrossRef] [PubMed]

20. Aydemir, G.; Kasiri, Y.; Bartók, E.M.; Birta, E.; Fröhlich, K.; Böhm, V.; Mihaly, J.; Rühl, R. Lycopene supplementation restores vitamin A deficiency in mice and possesses thereby partial pro-vitamin A activity transmitted via RAR signaling. Mol. Nutr. Food Res. 2016, 60, 2413-2420. [CrossRef]

21. Di Pietro, N.; Di Tomo, P.; Pandolfi, A. Carotenoids in cardiovascular disease prevention. JSM Atheroscler. $2016,1,1$.

22. Bai, S.; Tao, R.; Yin, L.; Ni, J.B.; Yang, Q.S.; Yan, X.H.; Yang, F.; Guo, X.P.; Li, H.X.; Teng, Y.W. Two B-box proteins, PpBBX18 and PpBBX21, antagonistically regulate anthocyanin biosynthesis via competitive association with Pyrus pyrifolia ELONGATED HYPOCOTYL 5 in the peel of pear fruit. Plant J. 2019, 100, 1208-1223. [CrossRef] [PubMed]

23. Xu, P.; Christopher, Z.; Li, Y.; Wu, J.; Liu, L.; Liu, Z.; Cai, R.; Lian, H. Transcriptome sequencing reveals role of light in promoting anthocyanin accumulation of strawberry fruit. Plant Growth Regul. 2018, 86, 121-132. [CrossRef]

24. Yamagishi, M. A novel R2R3-MYB transcription factor regulates light-mediated floral and vegetative anthocyanin pigmentation patterns in Lilium regale. Mol. Breed. 2016, 36, 1. [CrossRef] 
25. Meir, S.; Kochanek, B.; Glick, A. Reduced Petal Pigmentation in Lisianthus (Eustoma grandiflorum) Flowers under low light conditions is associated with decreased expression of anthocyanin biosynthesis genes. Acta Hortic. 2010, 877, 1735-1744. [CrossRef]

26. Meng, X.; Peng, J.; Wang, X. Anthocyanin Accumulation and CHS, DFR Gene expression regulated by light and sugar in Gerbera hybrida Ray Floret. Acta Hortic. Sin. 2007, 34, 227-230.

27. Wang, A.; Li, R.; Ren, L.; Gao, X.; Zhang, Y.; Ma, Z.; Ma, D.; Luo, Y. A comparative metabolomics study of flavonoids in sweet potato with different flesh colors (Ipomoea batatas (L.) Lam). Food Chem. 2018, 260, 124-134. [CrossRef]

28. Chen, W.; Gong, L.; Guo, Z.; Wang, W.; Zhang, H.; Liu, X.; Yu, S.; Xiong, L.; Luo, J. A novel integrated method for large-scale detection, identification, and quantification of widely targeted metabolites: Application in the study of rice metabolomics. Mol. Plant 2013, 6, 1769-1780. [CrossRef]

29. Dong, T.; Han, R.; Yu, J.; Zhu, M.; Zhang, Y.; Gong, Y.; Li, Z. Anthocyanins accumulation and molecular analysis of correlated genes by metabolome and transcriptome in green and purple asparaguses (Asparagus officinalis, L.). Food Chem. 2019, $271,18-28$. [CrossRef] 